\title{
Fragestellung präzisiert
}

Swiss Medical Board

Zum Bericht «Orale oder parenterale Behandlung des Eisenmangels» sind im Rahmen der Post-Publication-Review insbesondere Stellungnahmen zur Klarheit der Fragestellung eingegangen. Das Swiss Medical Board hat deshalb für den im Oktober 2014 veröffentlichten Bericht die Fragestellung klarer formuliert.

Nach Veröffentlichung des Berichts «Orale oder parenterale Behandlung des Eisenmangels» lud die Trägerschaft des Swiss Medical Board alle interessierten Stellen zu Stellungnahmen im Rahmen einer Post-Publication-Review ein. Die Mehrzahl der eingegangenen Reaktionen hat darauf hingewiesen, dass aus dem Bericht die zugrunde liegende Diagnose bzw. die betroffene Patientengruppe nicht klar hervorgehe. Zudem wurde moniert, dass der Begriff "symptomatischer, schwerer Eisenmangel» zu ungenau definiert sei.

Der Bericht analysiert die Behandlung des symptomatischen, schweren Eisenmangels, dessen Symptome hauptsächlich markante Müdigkeit und Abgeschlagenheit einschliessen. Dabei geht es um die Frage, ob die parenterale (intravenöse) Therapie gegenüber einer oralen Therapie für den Patienten hinsichtlich Wirksamkeit und Lebensqualität Vorteile bringt.

Die Behandlung des Eisenmangels ohne Symptome ist nicht Gegenstand des Berichts. Auch auf eine präzise Definition des «schweren Eisenmangels» verzichtet der Fachbericht, weil die Definition nicht an allgemeingültigen Laborwerten festgemacht werden kann. Der Fachbericht geht jedoch davon aus, dass ein schwerer Eisenmangel die Stadien II «Eisenmangel mit beginnender Beeinträchtigung der Blutbildung ( $\mathrm{Hb}$ normal, MCV und MCH erniedrigt)» und III «Eisenmangelanämie (Hb erniedrigt)" umfasst. Weiter beschränkt sich der Bericht auf die Form der Therapie günstigere, einfache orale oder teurere intravenöse Verabreichung -, wenn die Indikation zur Substitution unbestritten ist. Die Indikation zur Behandlung des Eisenmangels wird nicht untersucht.

\section{Schlussfolgerungen bleiben unverändert}

Die Rückmeldungen aus der Ärzteschaft haben die Expertengruppe dazu veranlasst, den Bericht in Bezug auf die angesprochenen Punkte zu revidieren. Der Fokus auf "symptomatischen, schweren Eisenmangel» geht neu bereits aus dem Titel hervor. Die Schlussfolgerungen und Empfehlungen im Bericht bleiben jedoch unverändert:

- Bei Patienten mit einem symptomatischen, schweren Eisenmangel oder einer Eisenmangelanämie ist nach sorgfältiger Abklärung eine parenterale Substitutionstherapie sinnvoll. Bei der Durchführung sind eine entsprechende Überwachung und Interventionsbereitschaft in Hinsicht auf eventuelle Nebenwirkungen sicherzustellen.

- Gleichzeitig ist eine geeignete Behandlung der Ursache(n) des Eisenmangels einzuleiten.

- Darüber hinaus sollte immer geprüft werden, ob Patienten im Zusammenhang mit einer chirurgischen Intervention von einer parenteralen Eisensubstitutionstherapie profitieren können.

Der Bericht zur Post-Publication-Review und der revidierte Fachbericht sind auf der Webseite des SMB veröffentlicht. 